Original Research Paper

\title{
Electrical Properties of Cobalt Oxide/Silica Nanocomposites Obtained by Sol-Gel Technique
}

\author{
${ }^{1,2}$ Gomaa A.M. Ali, ${ }^{3}$ Osama A. Fouad and ${ }^{4,5}$ Salah A. Makhlouf \\ ${ }^{I}$ Department of Chemsitry, Faculty of Science, Al-Azhar University, Assiut, Egypt \\ ${ }^{2}$ Faculty of Industrial Sciences and Technology, University Malaysia Pahang, Kuantan, Malaysia \\ ${ }^{3}$ Central Metallurgical Research and Development Institute, CMRDI, Helwan, Egypt \\ ${ }^{4}$ Department of Physics, Faculty of Science, Assiut University, Assiut, Egypt \\ ${ }_{5}^{5}$ Deanship of Scientific Research, Al Imam Mohammad Ibn Saud Islamic University (IMSIU), Riyadh, Saudi Arabia
}

Article history

Received: 17-10-2015

Revised: 21-10-2015

Accepted: 14-11-2015

Corresponding Author:

Salah A. Makhlouf

Department of Physics, Faculty of

Science, Assiut University, Assiut,

Egypt and

Deanship of Scientific Research,

Al Imam Mohammad Ibn Saud

Islamic University (IMSIU),

Riyadh, Saudi Arabia

Email:smakhlouf@gmail.com

\begin{abstract}
This work is an extension of our previous studies on cobalt oxide nanoparticles impeded in a silica matrix. Here we study the preparation and characterization of high cobalt content materials (60-90 wt\%). In addition, the DC electrical conductivity of the prepared materials in a wide temperature range (350-673 K) was measured and discussed. The activation energy has been obtained according to Mott's Small-Polaron Hopping (SPH) and Mott's and Greaves Variable Range Hopping (VRH) models.
\end{abstract}

Keywords: Sol-Gel, Cobalt Oxide/Silica, Nanocomposites, Electrical Conductivity

\section{Introduction}

Different preparation methods were reported for cobalt oxide nanoparticles embedded in a silica matrix such as sol-gel (Santos et al., 2012; Cantalini et al., 2005), hydrothermal (Kogelbauer et al., 1995; Bhoware and Singh, 2007), grafting, immobilization (Bhoware and Singh, 2007) and direct solution impregnation (Ming and Baker, 1995) methods. Among all of these techniques, sol-gel is considered as the most effective one producing mesoporous structures and fine particles (Cantalini et al., 2005; Fouad et al., 2011; Santos et al., 2012). Cobalt silica composite materials have been widely studied for many technological applications such as catalysis (Ming and Baker, 1995), humidity and gas sensing (Fouad et al., 2012; Cantalini et al., 2005), super capacitance (Ali et al., 2014). Cobalt oxide is an active material having multi oxidation states and silica has a porous structure, so the composite of cobalt oxide and silica would pocess interesting properties. Silica was found to be an excellent host material which offers homogenous distribution of the guest particles and prevents particle's growth and leading to the formation of highly dispersed nanocomposite materials.
Two main models have been reported to study the conductivity in transition metal oxide: Small-Polaron Hopping (SPH) and Variable Range Hopping (VRH) models at high and low temperatures, respectively (Ali et al., 2013; Hazra et al., 1995; Yildiz et al., 2009). Where, the electrical conduction takes place by the hopping movement of small polarons between two different oxidation states of the transition metal ions at high temperatures.

This study aims to prepare materials with high content of cobalt oxide dispersed in a silica matrix and study their electrical properties. The conduction mechanisms at high and low temperature ranges are to be discussed.

\section{Materials and Methods}

The materials for this study have been prepared by sol-gel method. Alcoholic solutions of cobalt nitrate and TEOS were used as cobalt and silica source, respectively. The detailed experimental procedure has been reported in our previous reports (Ali et al., 2013; Fouad et al., 2012; 2011) and is described schematically in Fig. 1. 


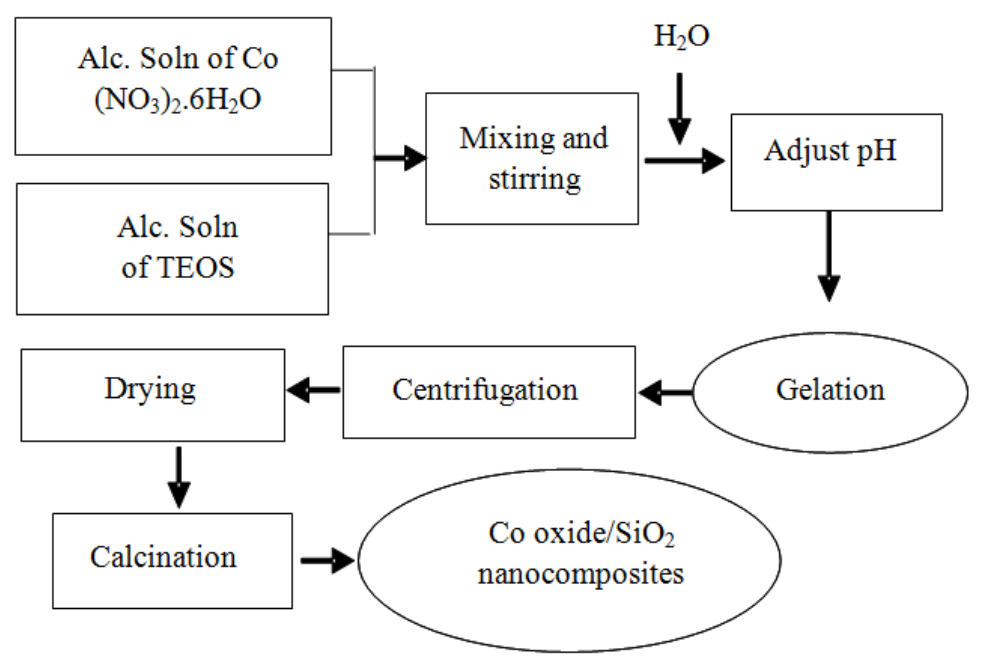

Fig. 1. Preparation scheme of cobalt oxide/silica nanocomposites via sol-gel method

Here high cobalt content samples have been prepared in which the cobalt weight content varies from 60 to $90 \%$. The codes SGO60, SGO80 and SGO90 were given for the composites containing 60,80 and $90 \%$ cobalt respectively.

The obtained phases were examined by X-Ray Diffraction (XRD; Philips PW1700 diffractometer, Netherlands) using $\mathrm{Cu}-\mathrm{K} \alpha$ radiation $(\lambda=0.15418 \mathrm{~nm})$ and a graphite monochromator in the $2 \theta$ range from 10 to $80^{\circ}$. The specific surface area, pore volume and pore size distribution were measured by $\mathrm{N}_{2}$ adsorptiondesorption technique (NOVA-3200, USA). The samples were degassed at $250^{\circ} \mathrm{C}$ for $3 \mathrm{~h}$ before analysis, the $\mathrm{N}_{2}$ isotherms were obtained at a temperature of $-196^{\circ} \mathrm{C}$. The specific surface area was calculated using the BrunauerEmmett-Teller (BET) method. The pore size distributions were determined by applying the Barrett-Joyner-Halenda (BJH) model to the nitrogen desorption branch.

Direct Current (DC) electrical measurements as a function of temperature were performed on the same pellets as described elsewhere (Ali et al., 2013; Fouad et al., 2012). The measurements were done in vacuum of $10^{-3}$ Torr in the temperature range of 300-673 K. A Keithley 617 electrometer operating at constant current mode was used for measuring the electrical resistance. A K-type thermocouple was used to measure the temperature using a Keithley 181 nanovoltmeter. All samples were preheated in vacuum up to $673 \mathrm{~K}$ and cooled down to $300 \mathrm{~K}$ at a rate of about $1 \mathrm{~K} \mathrm{~min}^{-1}$ during the measurements.

\section{Results and Discussion}

Figure 2 compares the XRD patterns of the cobalt oxide/silica composites. The XRD peaks were found to be related to $\mathrm{Co}_{3} \mathrm{O}_{4}$ (JCPDS card \#78-1970) and $\mathrm{CoSiO}_{3}$ (JCPDS card \#72-1508) phases. The peak broadening decrease with increasing the cobalt content indicating particles size increasing due to silica pore filling by cobalt species.

Further information about the structure morphology and porosity were obtained from $\mathrm{N}_{2}$ adsorptiondesorption measurements as shown in Fig. 3. BET specific surface $\left(S_{B E T}\right)$ area was found to be 63.7 and $28.8 \mathrm{~m}^{2} \mathrm{~g}^{-1}$ for SGO60 and SGO90 respectively. These high surface area value is due to the porous nature of silica matrix and fine cobalt oxide particles. In addition, SGO60 shows a pore diameter of $4 \mathrm{~nm}$ as presented in the inset of Fig. 3. The void fraction $(\varepsilon)$ was calculated using the relation $\varepsilon=V_{p} /\left(V_{p}+\left(1 / V_{p}\right)\right)$ where, $V_{P}$ is the pore volume. $\varepsilon$ was found to be 13.3 and $5.6 \%$ for SGO60 and SGO90, respectively. The particles size $\left(P S_{B E T}\right)$ could be obtained from BET data using the equation $P S_{B E T}=6000 /\left(S_{B E T} \times \rho\right)$, where $\rho$ is the material's density. SGO60 shows smaller particle size $(18 \mathrm{~nm})$ than SGO90 $(34 \mathrm{~nm})$. The specific surface area values for cobalt oxide embedded in silica matrix were found to be higher than those for pure cobalt oxide (Makhlouf et al., 2013) as a result of porous nature of silica which also giving lower particles size. All surface area parameters are listed in Table 1.

The electrical properties of the prepared materials have been investigated to study the conduction mechanism under wide temperature range. Figure 4 shows the variation of $\log$ $\sigma . T$ versus $1000 / T$ according to Equation 1:

$\sigma=\frac{\sigma_{o}}{T} \exp \left(-\frac{W}{K_{B} T}\right)$

where, $T$ is the absolute temperature, $K_{B}$ is the Boltzmann's constant, $W$ is the activation energy and $\sigma_{o}$ is the pre-exponential factor given as reported elsewhere (Ali et al., 2013). 
Gomaa A.M. Ali et al. / American Journal of Engineering and Applied Sciences 2016, 9 (1): 12.16 DOI: 10.3844/ajeassp.2016.12.16

Table 1. Calculated parameters for the cobalt oxide/silica nanocomposites

\begin{tabular}{lllllll}
\hline Sample & Co content $(\mathrm{wt} \%)$ & $S_{B E T}\left(\mathrm{~m}^{2} \mathrm{~g}^{-1}\right)$ & $P S_{B E T}(\mathrm{~nm})$ & $\varepsilon(\%)$ & $W(\mathrm{eV})$ & $W_{H}(\mathrm{eV})$ \\
\hline SGO60 & 60 & 63.7 & 18 & 13.3 & 1.03 & 0.38 \\
SGO80 & 80 & ---- & ---- & --- & 1.33 & 0.36 \\
SGO90 & 90 & 28.8 & 34 & 5.6 & 1.34 & 0.30 \\
\hline
\end{tabular}

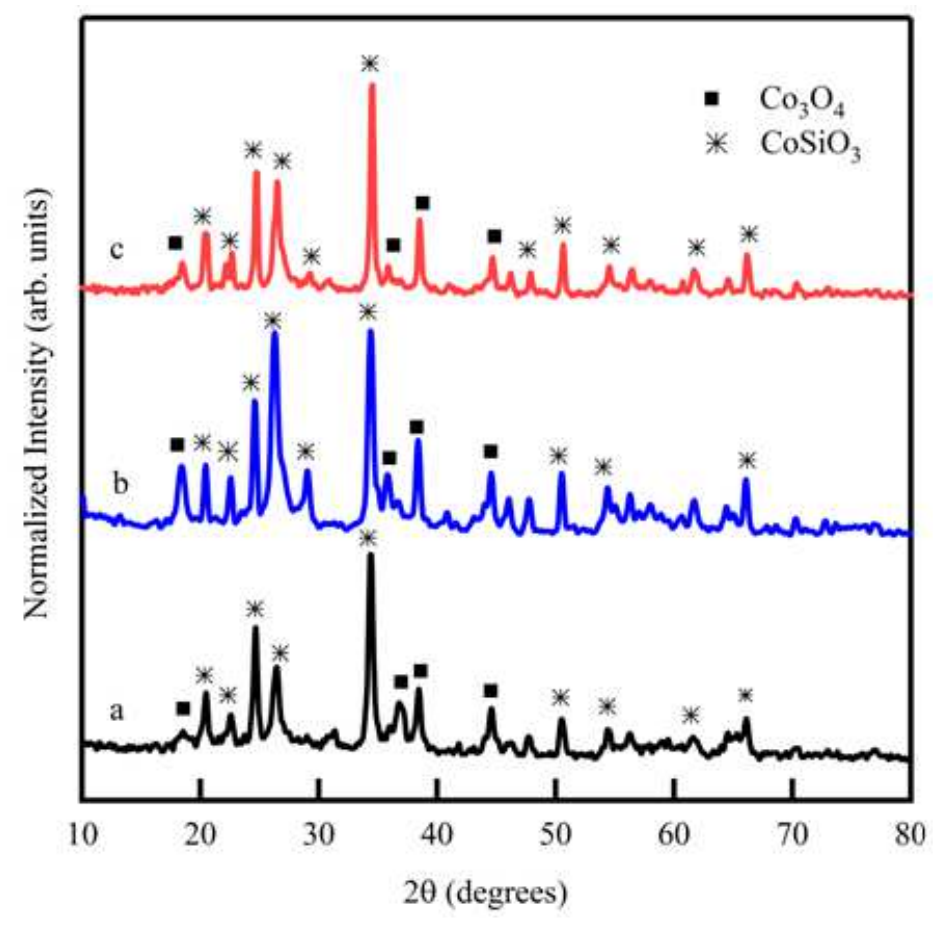

Fig. 2. XRD patterns for SGO60 (a), SGO80 (b) and SGO90 (c) nanocomposites

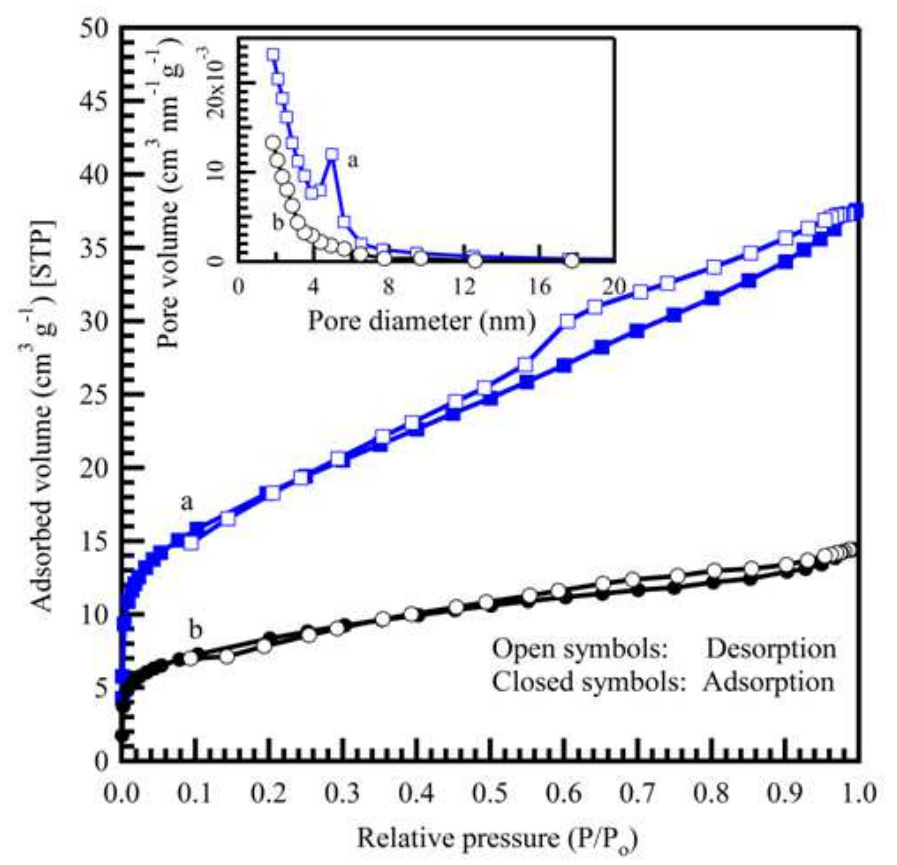

Fig. 3. $\mathrm{N}_{2}$ adsorption-desorption isotherms and pore size distribution (inset) for SGO60 (a) and SGO90 (b) nanocomposites 


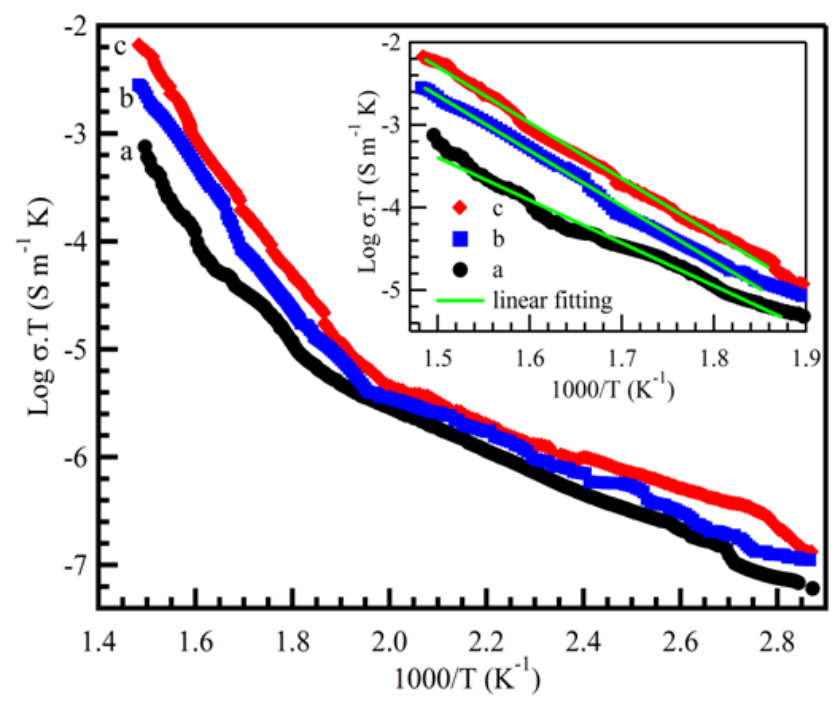

Fig. 4. Temperature variation of the DC conductivity of SGO60 (a), SGO80 (b) and SGO90 (c): Solid lines are the linear fits

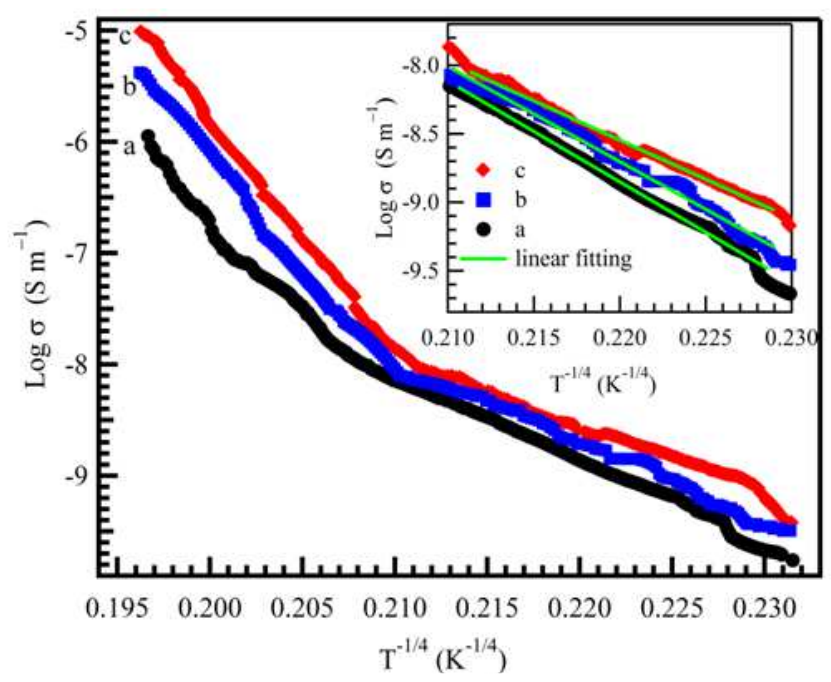

Fig. 5. Dependence of $\log \sigma$ on $\mathrm{T}^{-1 / 4}$ for SGO60 (a), SGO80 (b) and SGO90 (c): Solid lines are the linear fits

It is clear, the conductivity increases linearly with increasing temperature for all materials. In the higher temperature range (510-673 K), as shown in the inset of Fig. 4, the conductivity was found to be linearly depends on the reciprocal of temperature for all materials. This indicates the conduction is governed by SPH model. The electron-site interaction $\left(W_{H}\right)$ and static disorder $\left(W_{D}\right)$ which make-up the activation energy could be obtained as $W=W_{H}+1 / 2 W_{D}$ for $T>\theta_{D} / 2$ and $W=W_{D}$ for $T>\theta_{D} / 4$ (where, $\theta_{D}$ is Debye temperature). The activation energy was obtained from the slope and is listed in Table 1 together with other electrical parameters.

On the other hand, in the lower temperature range (350-510 K), it was found that the conductivity linearly depended on the $\left(\mathrm{T}^{-1 / 4}\right)$ according to Equation 2. Figure 5 shows the relation between $\log \sigma$ and $\left(T^{1 / 4}\right)$, the linear fitting is shown in the inset of Fig. 5. It is concluded that, the conductivity in this temperature region varies according to VRH model:

$\sigma=A^{\prime} \exp \left[\left(-\frac{T_{o}}{T}\right)^{1 / 4}\right]$

where, $A$ and $T_{o}$ are constants.

Figure 6 summarizes the dependence of the electrical conductivity on both temperature and cobalt content. It is clear that the conductivity of the materials under study is in the order of $\mathrm{SGO} 90>\mathrm{SGO} 80>\mathrm{SGO} 60$ which is in accordance with the cobalt content in the composite due to increasing charge carriers. At $650 \mathrm{~K}$, the conductivity of SGO90, SGO80 and SGO60 was $4.7 \times 10^{-6}, 2.0 \times 10^{-6}$ and $4.5 \times 10^{-6} \mathrm{~S} \mathrm{~m}^{-1}$, respectively. 


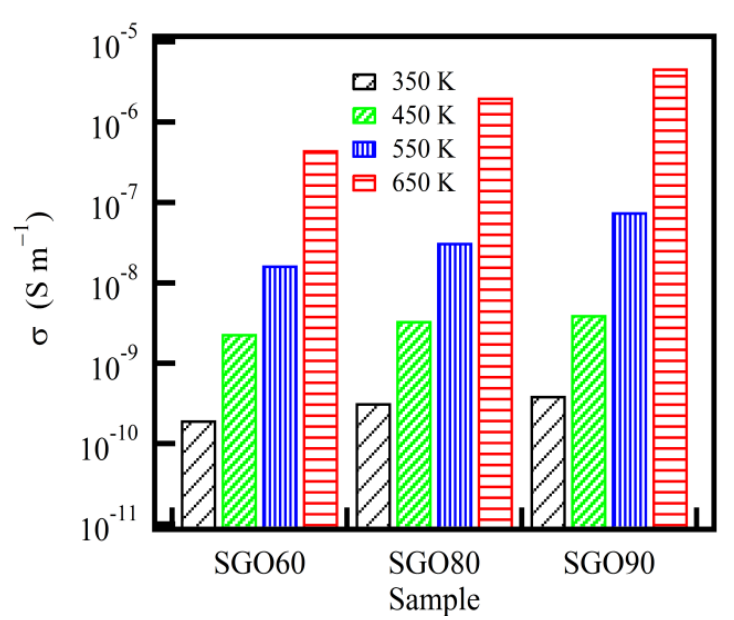

Fig. 6. $\log \sigma$ as a function of Co content, at indicated temperatures

\section{Conclusion}

Cobalt oxide/silica nanocomposites have been prepared via sol-gel method. Series of cobalt rich samples have been characterized using XRD and $\mathrm{N}_{2}$ adsorption-desorption techniques. Specific surface area was found to be $63.8 \mathrm{~m}^{2} \mathrm{~g}^{-1}$ with particle size of $18 \mathrm{~nm}$ for the composite containing $60 \mathrm{wt} \%$ cobalt. The DC electrical conductivity as a function of temperature was studied. The findings showed that the conduction mechanism is governed by small polaron hopping and variable range hopping models at high and low temperatures, respectively.

\section{Author's Contributions}

Gomaa A.M. Ali: $\mathrm{He}$ has conducted the experimental work and wrote the manuscript draft.

Osama A. Fouad: He has contributed in data analysis, discussion of the obtained results and revising the manuscript.

Salah A. Makhlouf: He has contributed in data analysis, discussion of the obtained results and revising the manuscript.

\section{Ethics}

This article is original and contains unpublished material. The corresponding author confirms that all of the other authors have read and approved the manuscript and no ethical issues involved.

\section{References}

Ali, G.A.M., O.A. Fouad and S.A. Makhlouf, 2013. Structural, optical and electrical properties of sol-gel prepared mesoporous $\mathrm{Co}_{3} \mathrm{O}_{4} / \mathrm{SiO}_{2}$ nanocomposites. J. Alloys Compounds, 579: 606-611.

DOI: 10.1016/j.jallcom.2013.07.095
Ali, G.A.M., O.A. Fouad, S.A. Makhlouf, M.M. Yusoff and K.F. Chong, 2014. $\mathrm{Co}_{3} \mathrm{O}_{4} / \mathrm{SiO}_{2}$ nanocomposites for supercapacitor application. J. Solid State Electrochem., 18: 2505-2512. DOI: $10.1007 / \mathrm{s} 10008-014-2510-3$

Bhoware, S.S. and A.P. Singh, 2007. Characterization and catalytic activity of cobalt containing MCM-41 prepared by direct hydrothermal, grafting and immobilization methods. J. Molecular Catalysis A, 266: 118-130. DOI: 10.1016/j.molcata.2006.09.031

Cantalini, C., M. Post, D. Buso, M. Guglielmi and A. Martucci, 2005. Gas sensing properties of nanocrystalline $\mathrm{NiO}$ and $\mathrm{Co}_{3} \mathrm{O}_{4}$ in porous silica solgel films. Sensors Actuators B, 108: 184-192. DOI: $10.1016 /$ j.snb.2004.11.073

Fouad, O.A., G.A.M. Ali, M.A.I. El-Erian and S.A. Makhlouf, 2012. Humidity sensing properties of cobalt oxide/silica nanocomposites prepared via solgel and related routes. Nano, 7: 1250038-1250047. DOI: $10.1142 / \mathrm{S} 1793292012500385$

Fouad, O.A., S.A. Makhlouf, G.A.M. Ali and A.Y. El-Sayed, 2011. Cobalt/silica nanocomposite via thermal calcination-reduction of gel precursors. Mater. Chem. Phys., 128: 70-76. DOI: 10.1016/j.matchemphys.2011.02.072

Hazra, S., A. Ghosh and D. Chakravorty, 1995. Electrical properties of sol-gel derived semiconducting cobalt silicate gel-glass. Solid State Commun., 96: 507-510. DOI: 10.1016/0038-1098(95)00369-X

Kogelbauer, A., J.C. Weber and J.G. Goodwin, 1995. The formation of cobalt silicates on $\mathrm{Co} / \mathrm{SiO}_{2}$ under hydrothermal conditions. Catalysis Lett., 34: 259-267. DOI: 10.1007/BF00806875

Makhlouf, S.A., Z.H. Bakr, K.I. Aly and M.S. Moustafa, 2013. Structural, electrical and optical properties of $\mathrm{Co}_{3} \mathrm{O}_{4}$ nanoparticles. Superlattices Microstructures, 64: 107-117. DOI: $10.1016 /$ j.spmi.2013.09.023

Ming, H. and B.G. Baker, 1995. Characterization of cobalt Fischer-Tropsch catalysts I. Unpromoted cobalt-silica gel catalysts. Applied Catalysis A, 123: 23-36. DOI: 10.1016/0926-860X(94)00220-7

Santos, G.A., C.M.B. Santos, S.W. Da Silva, E.A. Urquieta-González and P.P.C. Sartoratto, 2012. Solgel synthesis of silica-cobalt composites by employing $\mathrm{Co}_{3} \mathrm{O}_{4}$ colloidal dispersions. Colloids Surfaces A, 395: 217-224. DOI: $10.1016 /$ j.colsurfa.2011.12.033

Yildiz, A., S.B. Lisesivdin, M. Kasap and D. Mardare, 2009. Non-adiabatic small polaron hopping conduction in Nb-doped $\mathrm{TiO}_{2}$ thin film. Physica B, 404: 1423-1426. DOI: 10.1016/j.physb.2008.12.034 